\title{
Ultrasound-guided arterial catheterization
}

\section{Sung-Ae Cho*, Young-Eun Jang*, Sang-Hwan Ji, Eun-Hee Kim, Ji-Hyun Lee, Hee-Soo Kim, and Jin-Tae Kim}

Received February 1, 2021

Revised March 4, 2021

Accepted March 14, 2021
Department of Anesthesiology and Pain Medicine, Seoul National University Hospital, Seoul National University College of Medicine, Seoul, Korea

\section{Corresponding author}

Jin-Tae Kim, M.D., Ph.D.

Department of Anesthesiology and

Pain Medicine, Seoul National

University Hospital, Seoul National

University College of Medicine, 101

Daehak-ro, Jongno-gu, Seoul 03080,

Korea

Tel: 82-2-2072-3295

Fax: 82-2-747-8364

E-mail: jintae73@gmail.com

*Sung-Ae Cho and Young-Eun Jang contributed equally as the first author of this study.
Ultrasonography facilitates arterial catheterization compared to traditional palpation techniques, especially in small arteries. For successful catheterization without complications, practitioners should be familiar with the anatomic characteristics of the artery and ultrasound-guided techniques. There are two approaches for ultrasound-guided arterial catheterization: the short-axis view out-of-plane approach and the long-axis view in-plane approach. There are several modified techniques and tips to facilitate ultrasound-guided arterial catheterization. This review deals with the anatomy relevant to arterial catheterization, several methods to improve success rates, and decrease complications associated with arterial catheterization.

Keywords: Anatomy; Artery; Catheterization; Ultrasonography.

\section{INTRODUCTION}

Arterial pressure monitoring is essential for the management of hemodynamically unstable patients. The placement of an arterial catheter is sometimes technically challenging because some arteries have small calibers, anatomic variations, or arterial calcifications, or vasospasms. Multiple cannulation attempts are associated with a higher failure rate and complications such as hematoma, hemorrhage, vasospasm, occlusion, and infection. Ultrasonography is a useful tool for peripheral arterial catheterization [1]. Ultrasound guidance has been shown to facilitate arterial catheterization with fewer attempts. Ultrasound guidance for arterial catheterization improves the first and second attempt success rates and decreases the rate of complications compared to palpation or Doppler auditory assistance [2]. In addition, ultrasonography is a good modality for evaluating the extent of the artery in vasospasm as well as relaxation of the spasm after treatment [3]. This review deals with the anatomy of arteries, several ultrasound-guided arterial cannulation techniques, as well as tips to improve success rates and decrease complications associated with arterial catheterization.

\section{ANATOMY}

\section{Upper extremity}

The radial, ulnar, and brachial arteries can be used for arterial catheterization of the upper extremity (Fig. 1). When an arterial catheter is placed at the upper extremity that is supplied with blood by the aberrant subclavian artery, it should be considered that arterial pressure may be underestimated by compression in its retroesophageal course. If the patient has an interrupted aortic arch, coarctation of the aorta, or Blalock-Tausig shunt, it should be

This is an Open Access article distributed under the terms of the Creative Commons Attribution Non-Commercial License (http://creativecommons.org/licenses/by-nc/4.0) which permits unrestricted non-commercial use, distribution, and reproduction in any medium, provided the original work is properly cited.

Copyright (C) the Korean Society of Anesthesiologists, 2021 


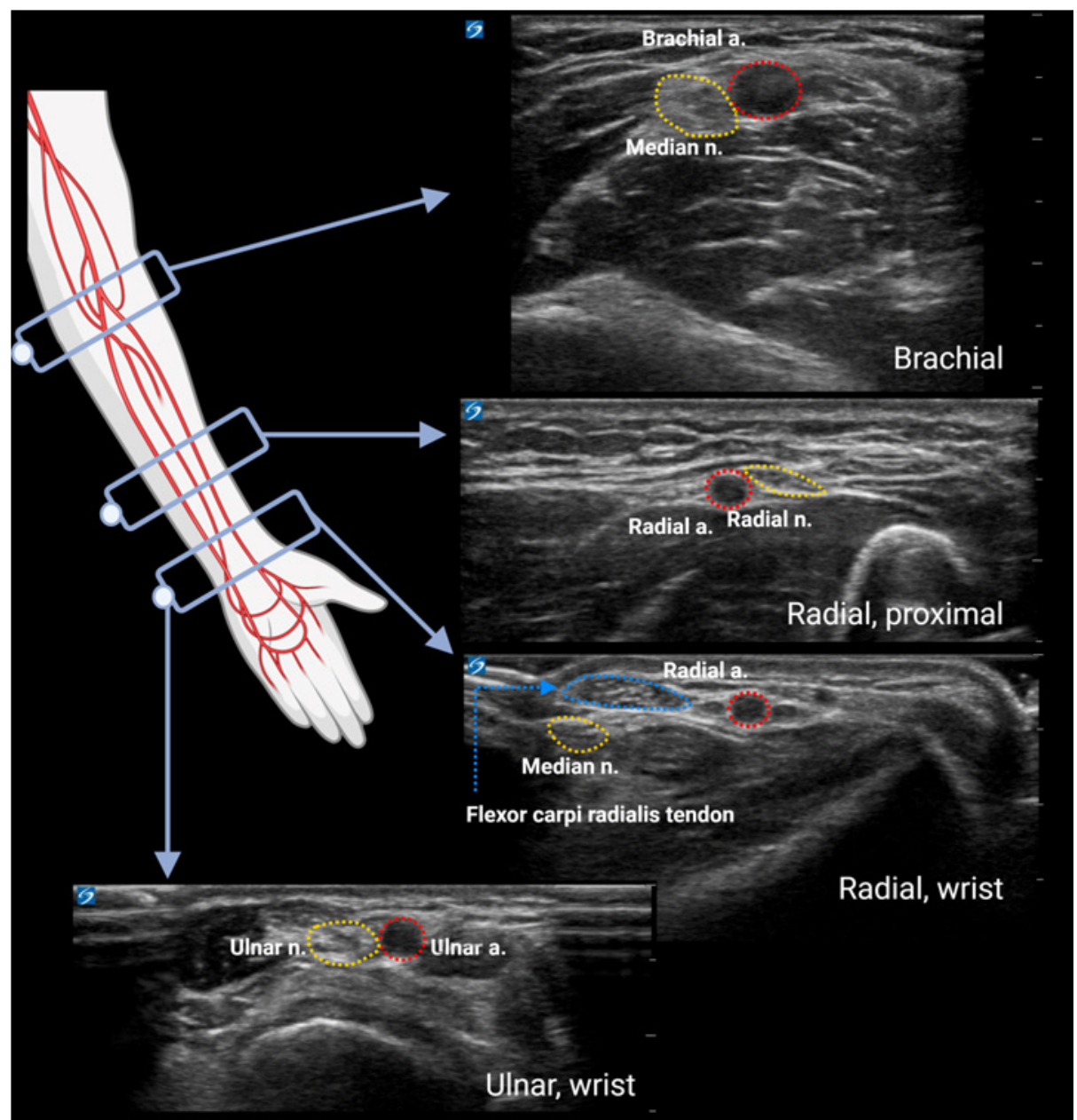

Fig. 1. Upper extremity visualized with schematic and sonographic images. The brachial artery is located in the middle of the upper extremity, while the median nerve is located on the medial side of the brachial artery. The radial artery and nerve run on the proximal side of the wrist but run separately in the distal part, while the median nerve is located near the radial artery at the distal wrist. The ulnar artery and nerve run together in the distal wrist. a.: artery, n.: nerve.

noted that the pressure measured from the upper extremity may not accurately represent the systemic arterial pressures at other sites.

\section{Radial artery}

The radial artery is the most commonly preferred site for arterial catheterization $[4,5]$ because of adequate collateral blood supply, low complication rates, and easy accessibility. The average diameter of the radial artery in adults and children is $2.2-2.3 \pm 0.4 \mathrm{~mm}[6,7]$ and $1.2-1.3 \pm 0.2 \mathrm{~mm}$ $[8,9]$, respectively. The average depth of the radial artery from the skin in adults and children is $2.5 \pm 0.3 \mathrm{~mm}$ [10] and $2.3 \pm 0.7 \mathrm{~mm}$ [8], respectively. The radial artery originates from the brachial artery and reaches the lateral side of the wrist [11]. After the radial artery divides into the superficial and deep branches at the styloid process level of the radial head, the radial and ulnar arteries are connected with the superficial palmar and deep palmar arches. As some cases reported absent or dual radial arteries [11], prescanning with ultrasound before radial arterial catheterization or real-time ultrasound-guided procedure is recommended [12]. Since the modified Allen's test has been debated as having low sensitivity to detect patency of collateral circulation [13], a recent guideline by the European Society of Anaesthesiology recommends performing the modified Allen's test using ultrasound and color Doppler before radial artery catheterization [12].

\section{Ulnar artery}

The ulnar artery is relatively smaller than the radial artery in the palpable region of the wrist, although it is larger than the radial artery at the division site just below the el- 
bow. The average diameter of the ulnar artery in adults is 2.3-2.5 $\pm 0.5 \mathrm{~mm}[6,7,14]$, which is smaller than the radial artery at the wrist level. The ulnar artery is a branch of the brachial artery and runs along the medial side of the wrist. The artery is located lateral to the ulnar nerve and flexor carpi ulnaris tendon in the palpable region of the wrist. Because the ulnar artery is also connected to the radial artery through the palmar arch, the modified Allen's test with ultrasound would be helpful in preventing vascular complications [12].

\section{Brachial artery}

Brachial arteries are usually not recommended for cannulation because of the absence of collateral flow. However, the brachial artery could be considered for cannulation in neonates and small children [15], The average diameter of the brachial artery above the antecubital fossa is $3.9 \pm$ $0.5 \mathrm{~mm}$ in adults [16] and $3.4 \pm 0.2 \mathrm{~mm}$ in children [17]. The palpable site of the brachial artery is on the medial side of the biceps tendon on the anterior part of the elbow. Usually, the median nerve runs together medially with the brachial artery on the elbow. It is vulnerable to damage when the blind technique is used for brachial artery cannulation.

\section{Lower extremity}

The dorsalis pedis, posterior tibial, and femoral arteries can be used for arterial catheterization of the lower extremities (Fig. 2).

\section{Dorsalis pedis artery}

The dorsalis pedis artery is representative of the arterial

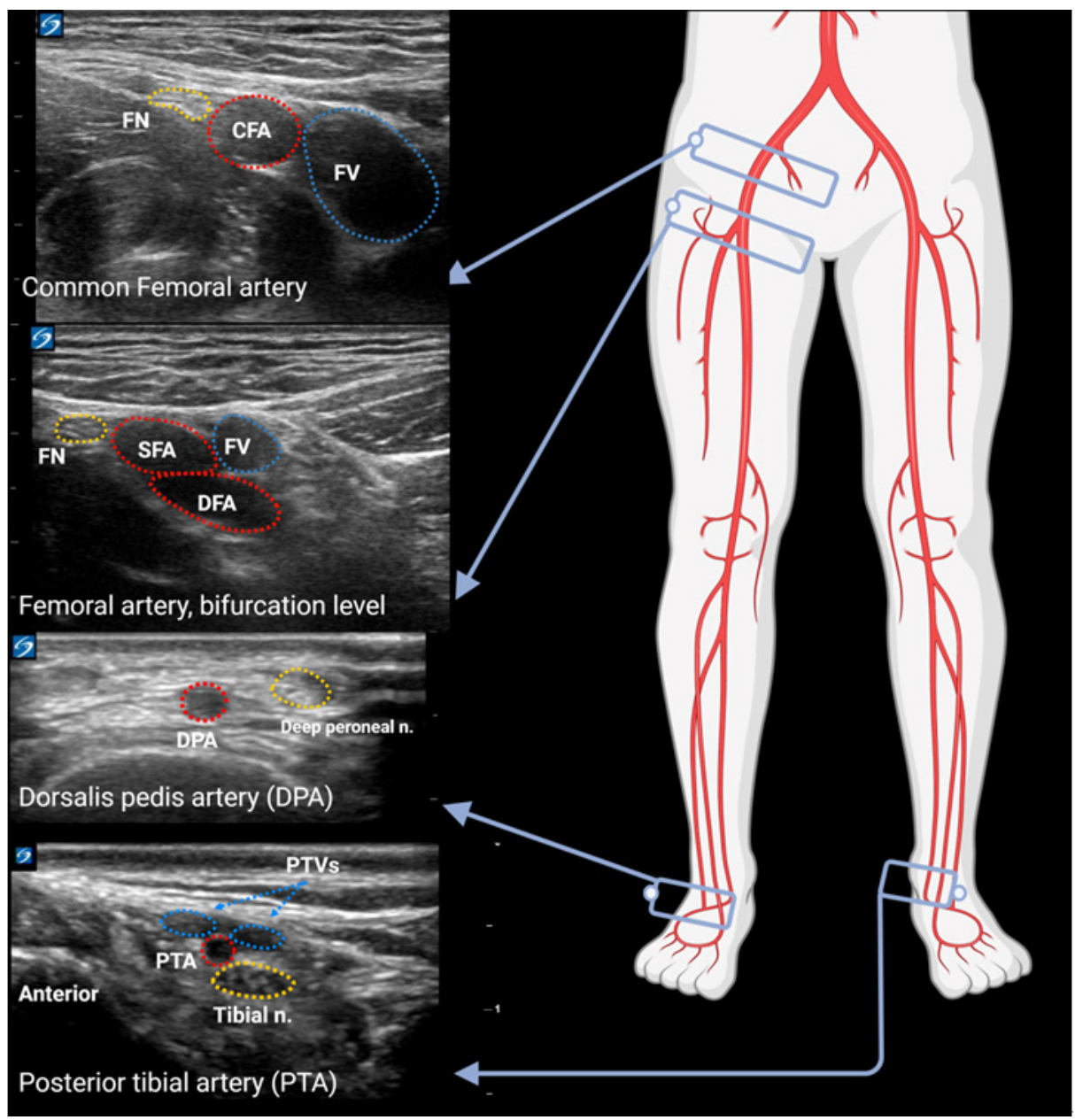

Fig. 2. Lower extremity visualized with schematic and sonographic images. The common femoral artery runs with the vein in the medial and the nerve in the lateral. In the slightly distal part, it divides into superficial femoral artery and deep femoral artery. The dorsalis pedis artery is located with the deep peroneal nerve on the medial side (anatomical variation), while the posterior tibial artery runs with the tibial nerve and veins. CFA: common femoral artery, DFA: deep femoral artery, FN: femoral nerve, FV: femoral vein, n.: nerve, PTV: posterior tibial vein, SFA: superficial femoral artery. 
cannulation site on the anterior side of the foot and originates from the anterior tibial artery. The diameter of the dorsalis pedis artery in adults and children is 3.0-3.6 \pm 1.2 $\mathrm{mm}$ [18] and 1.0-1.3 $\pm 0.2 \mathrm{~mm}[8,19]$, respectively. Its depth from the skin surface in children is $2.5 \pm 0.9 \mathrm{~mm}$ [8]. The landmark for its palpation is the most prominent part of the navicular bone and extensor hallucis longus tendon. The deep peroneal nerve is usually located lateral to the dorsalis pedis artery, with anatomical variations.

\section{Posterior tibial artery}

The posterior tibial artery is a reasonable alternative to the radial artery for ultrasound-guided arterial cannulation in small children [8]. The diameter of the posterior tibial artery is $3.1 \pm 0.4 \mathrm{~mm}$ [20] in adults and $1.4 \pm 0.3 \mathrm{~mm}$ in children [8]. Its depth from the skin surface is $3.4 \pm 1.2 \mathrm{~mm}$ in children [8], which is located deeper than other peripheral arteries. It originates from the popliteal artery and runs along the medial border of the fibula, along with the posterior tibial vein and tibial nerve. The most palpable site of the posterior tibial artery, called Pimenta's point, is found between the medial malleolus and the Achilles tendon. This artery is connected to the anterior tibial and peroneal arteries. Its depth from the skin surface decreases as the position of the ankle changes (e.g., dorsiflexion or eversion) [8]. Dorsiflexion of the ankle is helpful in posterior tibial arterial cannulation.

\section{Femoral artery}

The femoral artery is the largest accessible artery for arterial catheterization and continues from the external iliac and common iliac arteries. Because femoral arterial pressure represents the central arterial pressure, femoral arterial catheterization is useful in cardiopulmonary bypass $[21,22]$ or liver transplantation [23], when peripheral arterial pressure monitoring does not reflect central blood pressure. The average diameter of the common-, superficial-, and deep-femoral artery in adults is $6.6 \pm 1.2 \mathrm{~mm}, 5.2 \pm 1.2$ $\mathrm{mm}, 4.9 \pm 0.9 \mathrm{~mm}$ [24], respectively. The average diameter of the pediatric femoral artery is $2.8 \pm 0.8 \mathrm{~mm}$ in those aged $0-1$ months, and increases to $5.6 \pm 1.1 \mathrm{~mm}$ in those aged 5-7 years at $1 \mathrm{~cm}$ distal to the inguinal ligament [25]. The most palpable site of the femoral artery is under the inguinal ligament, between the anterior superior iliac crest and the pubic bone. Since the femoral artery is located between the femoral nerve (laterally) and femoral vein (medially) [26], femoral arterial catheterization with a blind technique is risky and increases complication rates. Therefore, ultrasound-guided femoral arterial catheterization is recommended in adults and children for safe and easier catheterization [12]. Moreover, as the overlap of the femoral vein increases by approximately $55 \%$ when it runs distal to the inguinal ligament, ultrasound-guided catheterization has been increasingly used [25]. External rotation of the hip joint can decrease femoral artery and vein overlapping. Femoral arterial catheterization should be performed carefully because serious complications, such as retroperitoneal hemorrhage or intra-abdominal organ injury, can occur.

\section{ULTRASOUND-GUIDED TECHNIQUES}

Ultrasound-guided arterial cannulation improves success rates and reduces procedural time and procedure-related complications compared with the direct pulse palpation method [1,12,27-29]. Ultrasound guidance is particularly useful when it is difficult to feel the arterial pulse, the arteries have small diameters (pediatric patients) or deep locations (obese patient), as well as in dehydration, hypotension, low cardiac output, non-pulsatile blood flow, vascular disease, and previously unsuccessful arterial cannulation $[12,27]$.

A successful first cannulation attempt is important $[9,30]$. Failed cannulation attempts can cause arterial vasospasm, intra-arterial or extra-arterial hematoma, and further decrease the overall success rate. Multiple cannulation attempts can cause serious complications such as nerve injury, pseudoaneurysm, total arterial occlusion, and permanent distal ischemia [12]. Therefore, pre-procedural sonographic evaluation should be performed to identify the artery with the largest diameter, optimal depth, and straight course to increase the first-attempt success rate.

A high-frequency linear transducer or a hockey stickshaped transducer is used to visualize the artery. A small hockey stick-shaped transducer helps to adjust the alignment of the needle and artery in pediatric patients. Duplex ultrasonography and color Doppler can be used to identify arterial pulses and to perform a modified Allen's test before radial artery cannulation [31,32].

After choosing the target artery, it is important to adjust the height of the table and the location of the ultrasound machine so that the operator can perform arterial cannulation in a comfortable position. The operator then optimizes the patient's wrist or ankle posture and fixes the joint so 
that cannulation can be performed without movement in the target artery. The depth and diameter of the target artery should be considered when selecting the size of the arterial catheter. Hand hygiene should be performed before gloving. An alcohol-based chlorhexidine antiseptic is recommended for skin preparation. When a sterile sheath is used, the transducer should be placed inside the sterile sheath carefully to prevent air from entering between the transducer and sheath.

There are two basic ultrasound-guided approaches: the short-axis view (SAX) out-of-plane approach and the long-axis view (LAX) in-plane approach. Moreover, some studies have proposed an oblique approach [20,33]. For radial artery cannulation, the currently available literature suggests similar success rates with either the SAX out-ofplane approach or the LAX in-plane approach in adults [34-36] and pediatric patients [37].

\section{Short axis view out-of-plane approach}

In the SAX out-of-plane approach, the transducer is placed perpendicular to the course of the target artery. The course and condition of the target artery can be checked by moving the transducer proximally and distally. In a good SAX, the target artery should appear as a pulsatile anechoic circle at the center of the screen. The needle is inserted approximately $30^{\circ}$ to $45^{\circ}$ to the skin and perpendicular to the center of the transducer. The middle mark on the ultrasound probe corresponding to the middle mark in the ultrasound image is helpful for inserting the catheter needle into the center of the target artery. The needle appeared as a hyperechoic dot and advanced until the anterior wall of the target artery collapses. If the needle tip is not visualized well, slight tiling or movement of the transducer will be helpful. When the needle enters the lumen of the artery, the collapsed anterior wall re-expands and flashback of the blood occurs (Fig. 3A, B).

The advantages of the SAX out-of-plane approach include: 1) easy scanning of the target artery, 2) easy maintenance of the transducer during the procedure, and 3) easy directing of the needle toward the target artery. The disadvantages of the SAX out-of-plane approach include 1) non-visualization of the entire course of the artery, 2) possibly not being able to visualize the needle tip on the ultrasound screen, and therefore, 3) cause possible puncture of the arterial posterior wall or misplacement of the needle tip behind the scene.

\section{Modified short axis view out-of-plane approach}

\section{The ultrasound-guided dynamic needle tip positioning technique}

The ultrasound-guided dynamic needle tip positioning technique has been proposed to overcome the shortcomings of the SAX out-of-plane approach while maintaining its advantages. The needle tip of the shaft is displayed as a hyperechoic dot on the screen, and this dot can be more easily identified by adjusting the angle of needle insertion into the skin. The needle tip can be identified in the SAX by moving and tracing the probe. After puncturing the anterior wall of the artery and confirming the hyperechoic dot in the vessel lumen, the probe is moved proximally until the needle tip disappears and then the needle is slightly advanced until the hyperechoic dot reappears. This procedure is repeated until the needle with the catheter is fully inserted into the artery. In this procedure, the angle of needle advancement should be lower than the insertion angle concerning the course of the artery so as not to puncture the posterior wall of the artery (Fig. 3C-E). This technique increased the first and overall success rates compared to palpation in both adults and children [38-40].

\section{Line on the probe for the midpoint}

This method is advantageous for aligning the center of the transducer with the needle insertion point and the center of the artery. A developing line tied to the midpoint of the ultrasound probe and perpendicular to the long axis may improve the success rate of cannula insertion into the radial artery on the first attempt [35]. Acoustic shadowing via the use of double developing lines significantly improved the success rate of radial artery puncture in young children compared with that achieved using traditional ultrasound guidance [41].

\section{Long axis view in-plane approach}

In the LAX in-plane approach, the transducer is placed parallel to the course of the target artery. First, the target artery is scanned in SAX to capture the artery at the center of the screen. Then, the transducer is rotated by $90^{\circ}$ while keeping the artery at the center of the screen. In a good LAX, the target artery should appear as a pulsatile tubular anechoic structure across the screen. The needle is inserted approximately $30^{\circ}$ to $45^{\circ}$ to the skin and perpendicular to the center of the longitudinally applied transducer. The 


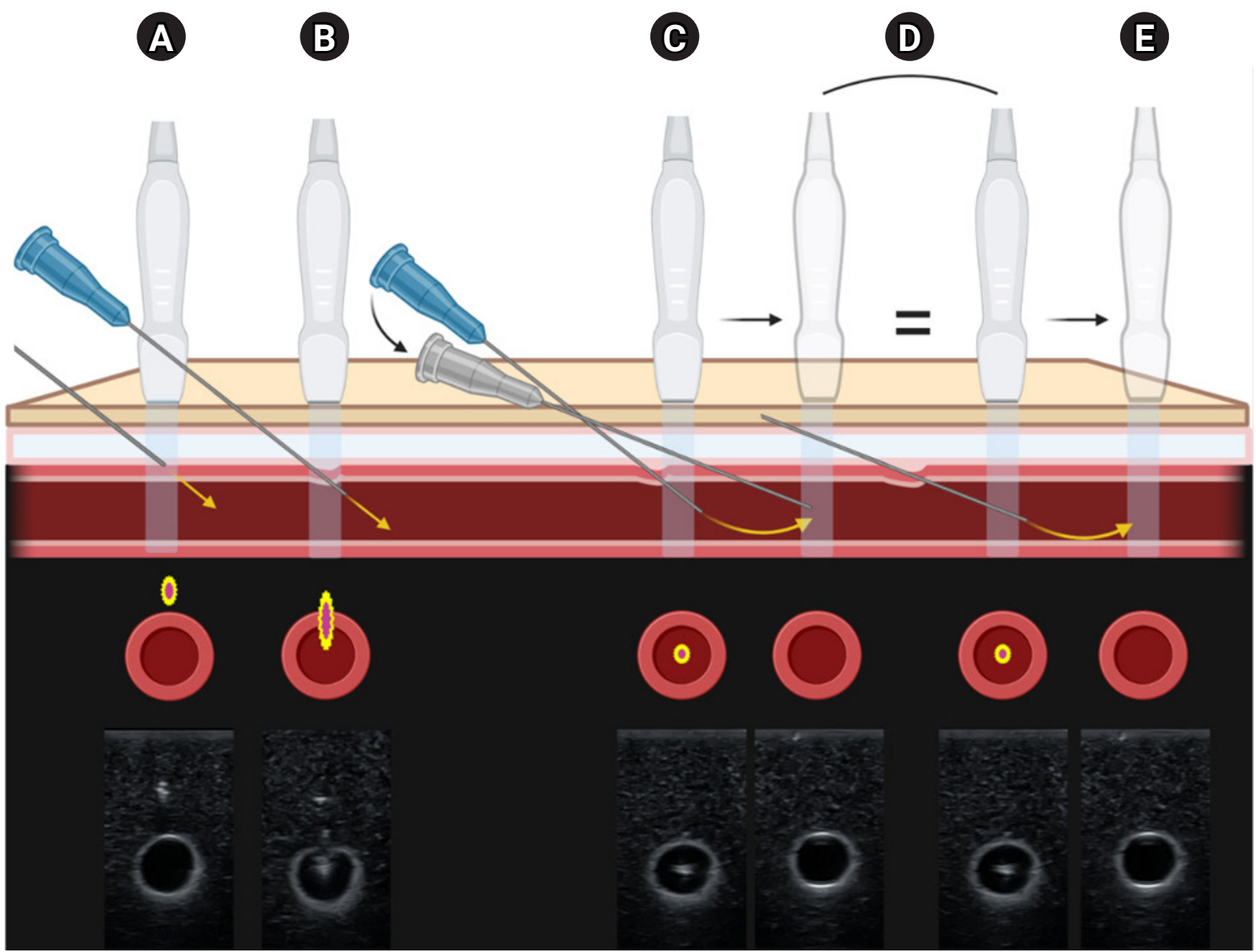

Fig. 3. Short axis view of the sonographic images in the vessel using the phantom model. (A) is the point at which the needle enters through the skin before entering the vessel. (B) is the point at which the needle first enters the vessel. When it is confirmed that the needle is in the vessel, like in (C), the ultrasound probe is dynamically moved to check the location of the needle tip as the catheter is advanced (D, E).

needle appears as a hyperechoic double line above the artery and is advanced until the anterior wall of the target artery is punctured. If the needle tip is not visualized well, slight tilting of the needle to the left or right would be helpful. When the needle tip enters the lumen of the artery, the collapsed anterior wall re-expands and flashbacks of the blood will occur (Fig. 4).

The major advantage of the LAX in-plane approach is that the course of the artery and needle tip can be observed throughout the procedure. Therefore, puncture of the posterior arterial wall or misplacement of the needle tip is less likely to occur. The disadvantages of the LAX in-plane approach include 1) difficulty in identifying whether the needle is on the right or left side of the artery and 2) difficulty in maintaining transducer position as well as alignment of the needle shaft, tip, and artery during the procedure. Although we assume that the image plane of the ultrasound machine is paper-thin, the ultrasound beam has a measurable thickness. This is called a slice-thickness artifact.
Therefore, the needle and target artery can appear on the screen in the LAX even though they are not exactly on the same plane [42]. Sometimes, the needle can be seen in the lumen of the target artery without a puncture. Therefore, it is important to confirm that the anterior wall of the target artery collapses during needle advancement. The LAX inplane approach requires a longer imaging time, but the total time to cannulation is not different from that of the SAX approach. The posterior wall puncture rate is lower in the LAX in-plane approach than in the conventional SAX outof-plane approach in children younger than 5 years of age [37].

\section{Oblique approach}

In the oblique approach, the transducer is placed parallel to the course of the target artery to obtain the image in the LAX. Then, the transducer is rotated from $10^{\circ}$ to $15^{\circ}$ while keeping the artery at the center of the screen $[20,33]$. 


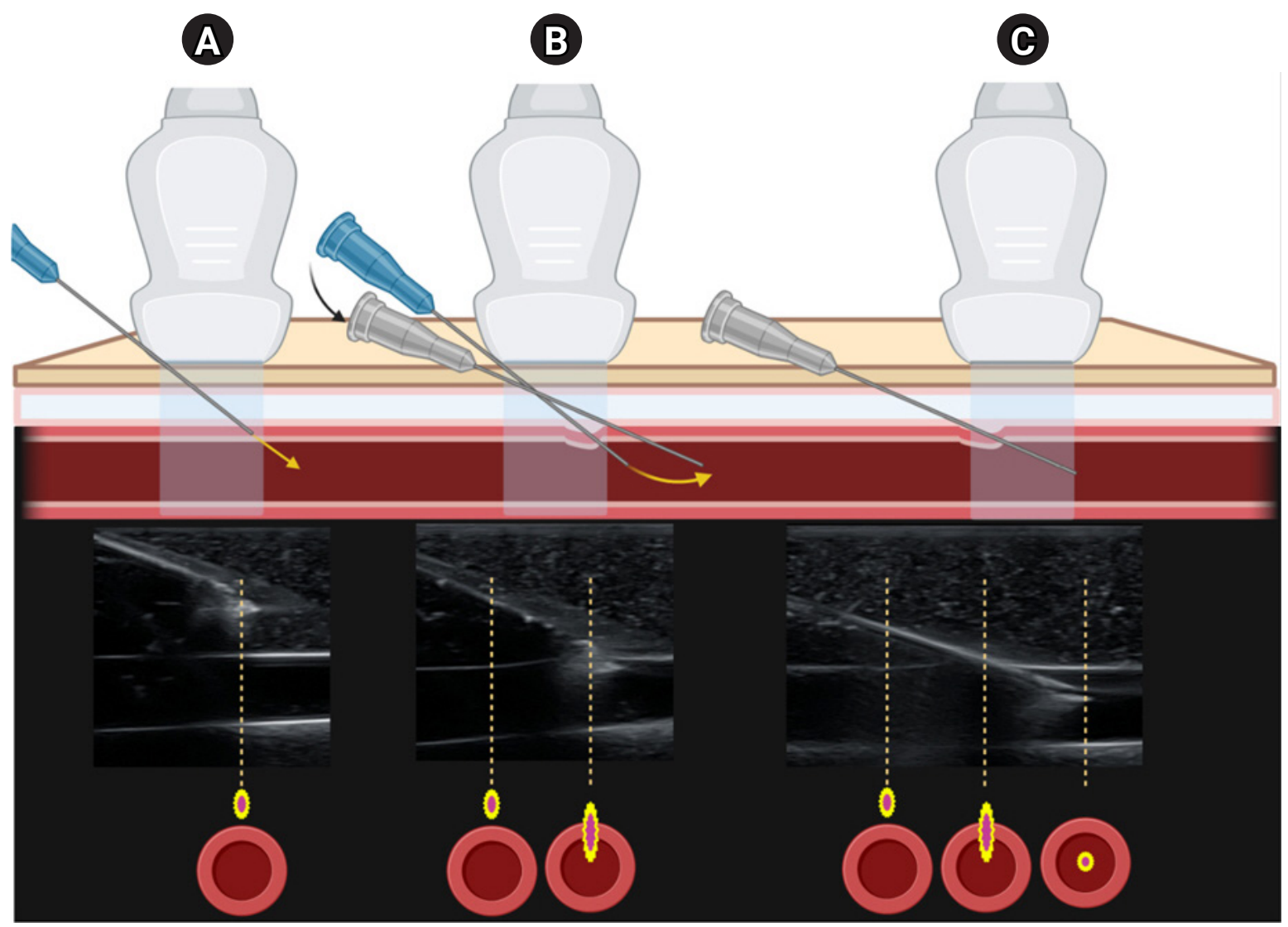

Fig. 4. Long-axis view of the sonographic images in the vessel using the phantom model. In the long-axis view, real-time visualization is possible before entering the vessel $(A)$, upon entering the vessel $(B)$, and after entering the vessel (C).

In a good oblique view, the target artery should appear as a pulsatile elliptical anechoic structure across the screen. The needle is inserted through the skin and the target artery similar to the LAX approach (Fig. 5). In adults, the oblique approach showed a higher first-attempt success rate and, shorter procedure time, and less vasospasm and hematoma than the SAX out-of-plane or LAX in-plane approach $[20,33,43]$. The advantages of the oblique approach are as follows: 1) ease of scanning the target artery and 2) visualization of the needle with fewer section-thickness artifacts.

\section{Other methods to improve success rates}

\section{Nitroglycerin}

Nitroglycerin dilates the radial artery and decreases the incidence of vasospasm [9,44-47]. In adult patients, nitroglycerin has been used for radial artery dilation by intra-arterial $[48,49]$, topical $[50,51]$, and subcutaneous administration $[44,46]$.

Application of topical nitroglycerin cream 30 min before radial artery cannulation has the advantage of being non-invasive and having significant vasodilatory effects on the radial artery in healthy adult patients $[50,51]$. Subcutaneous administration $(500 \mu \mathrm{g})$ is effective for localized vasodilation without causing systemic side effects $[44,46]$, and decreases the incidence of early radial artery occlusion after transradial coronary catheterization [47]. Radial artery cannulation can be difficult in infants and young children because of their small vessel sizes, with multiple attempts leading to vasospasm, hematoma, or a further decrease in the diameter of the artery. Subcutaneous nitroglycerin $(5 \mu \mathrm{g} / \mathrm{kg}$ in $0.5 \mathrm{ml})$ injection before radial artery cannulation improves the first-attempt success rate and reduces overall complication rates in pediatric patients younger than 2 years [9].

\section{Bevel orientation}

The orientation of the needle bevel can affect the ultrasound image of the needle tip when using the LAX in-plane approach. When the bevel-down approach is used, the needle tip is at the end of one long line on the image, while 


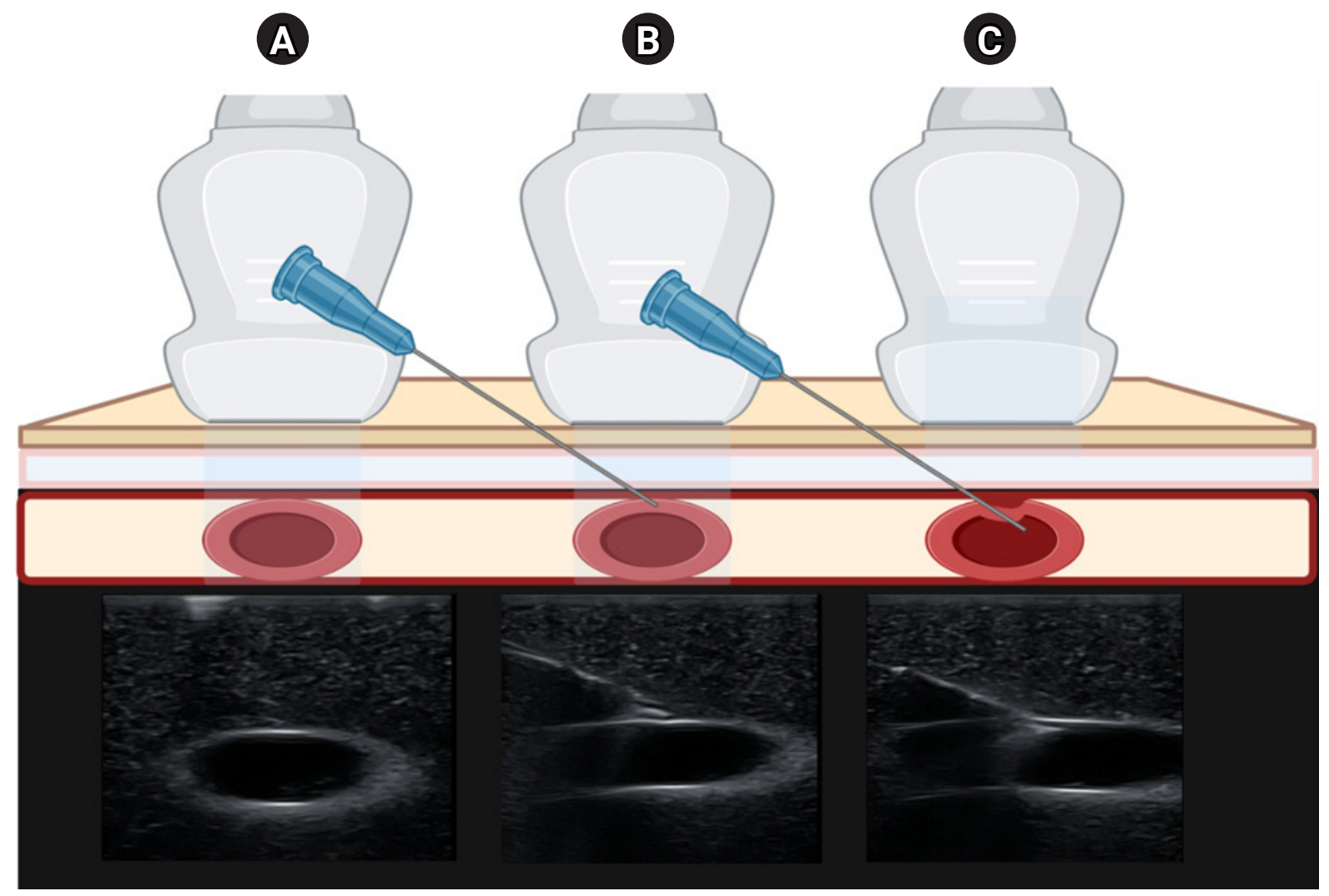

Fig. 5. Oblique view of the sonographic image in the vessel using the phantom model. In the oblique view, real-time visualization is possible before entering the vessel, which is oval in shape in the ultrasound image (A), upon entering the vessel (B), and after entering the vessel (C).

in the bevel-up approach, the needle tip is at the end of another short line called the "bevel line", which is observed in front and below the main long needle line. Neglecting to visualize the "bevel line" can result in inadvertent perforation of the posterior wall [52].

The bevel-down during ultrasound-guided SAX out-ofplane approach for radial artery catheterization exhibited a higher success rate with fewer complications compared to the bevel-up approach. Compared to the bevel-up approach, the bevel-down approach decreases posterior arterial wall injury in adults [10].

\section{Technique after the flashback of the blood}

There are two steps of arterial cannulation: puncture of the vessel with a needle and insertion of the catheter after the puncture. Even after successful puncture, advancement of the catheter is difficult in some cases. Possible causes include a non-optimal location of the needle tip or catheter tip and vasospasm. In such cases, the guidewire technique is useful (Fig. 6). For radial artery cannulation in neonates and infants, guidewire-assisted radial artery cannulation was superior to the direct technique in terms of first-at- tempt success rate and overall success rate without an increase in procedure time [30]. The use of a guidewire also increases the success rates in critically ill patients $[53,54]$.

\section{Wrist, ankle, and leg position for cannulation}

For radial artery cannulation, a recent meta-analysis showed that $45^{\circ}$ of wrist extension, compared to the neutral position, increased the anterior-posterior diameter of the radial artery and facilitated radial artery cannulation by increasing the success rate and decreasing the procedure time in adults $[55,56]$. There are no data regarding the effect of ankle position on the diameter, depth, or success rate of arterial catheterization in the dorsalis pedis artery or posterior tibial artery in adults.

In pediatric patients, Kim et al. [8] reported the effect of wrist and ankle position on the diameter and depth of the radial, posterior tibial, and dorsalis pedis arteries. Wrist extension up to $45^{\circ}$ decreased the depth of the radial artery without changes in diameter or cross-sectional area. For the posterior tibial artery, ankle dorsiflexion and eversion significantly decreased the depth of the posterior tibial artery from $5 \mathrm{~mm}$ to $4 \mathrm{~mm}$ from the skin, which could influ- 

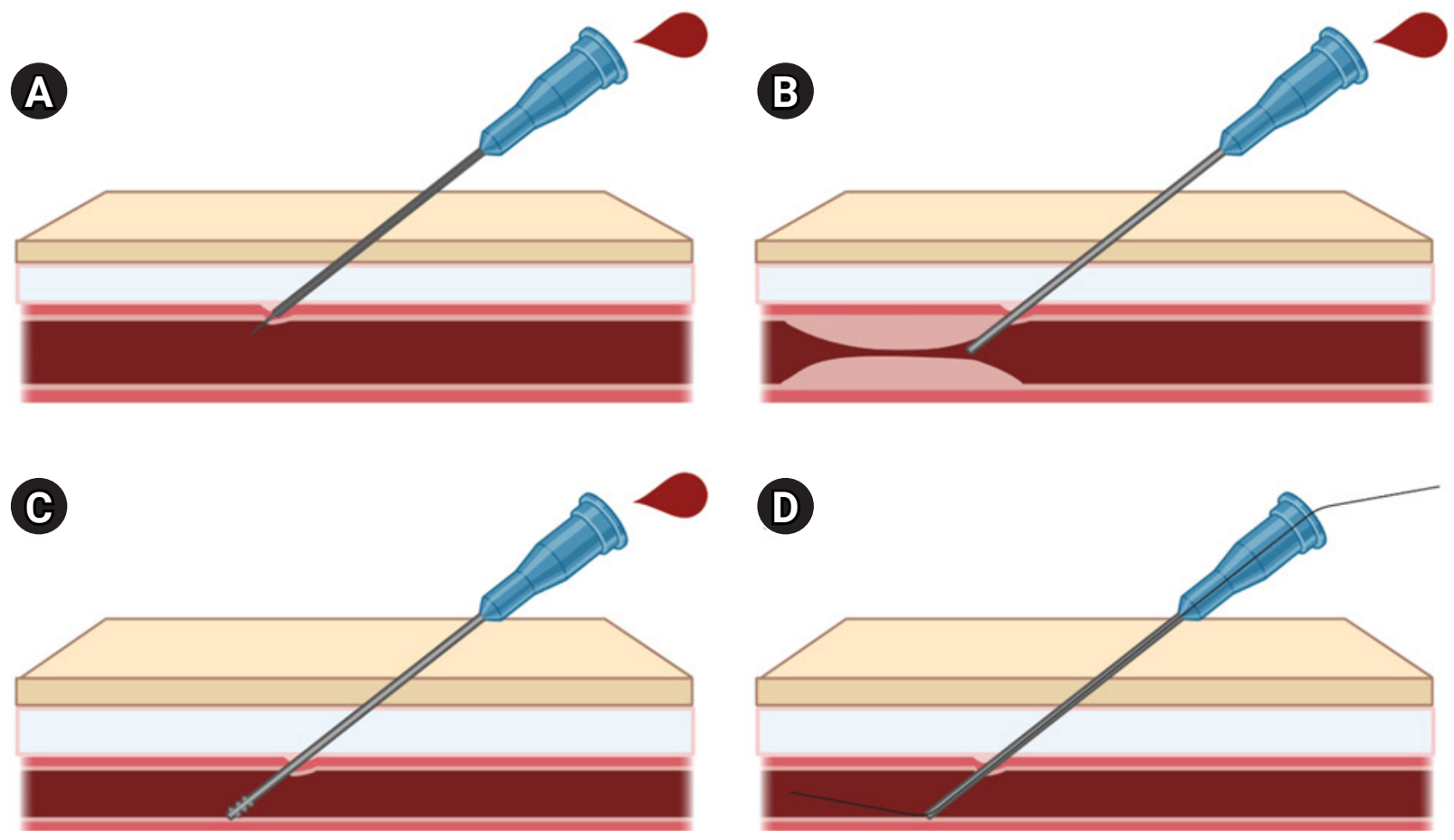

Fig. 6. Possible causes of failure in the advancement of the catheter after flashback of blood and its solutions. (A) The location of the catheter is not optimal because the needle tip spans the vessel wall and the inside the arterial lumen, but the catheter is outside the arterial lumen. (B) The catheter cannot be advanced due to vasospasm or intraluminal obstacles. (C) The catheter cannot be advanced because it gets stuck in the posterior wall of the blood vessel or is bent. (D) Guidewire insertion is helpful when the catheter or needle tip spans the vessel wall and inside the arterial lumen.

ence the success rate of posterior tibial arterial cannulation. For the dorsalis pedis artery, ankle plantar flexion decreased the cross-sectional area.

Overlapping of the common femoral artery and the femoral vein is observed in $65 \%$ of adults and $55 \%$ of pediatric patients $[25,57]$. Therefore, distinguishing the femoral artery and femoral vein is very important especially during the LAX approach. Arteriovenous fistula formation can occur after an inadvertent wall puncture between the femoral artery and femoral vein. In adults, external rotation and abduction of the leg (frog-leg position) [58] and a frog-leg position with a combination of back extension decreased the overlapping portion between the femoral artery and femoral vein [59]. In pediatric patients, leg abduction with a range between $48^{\circ}$ and $58^{\circ}$ and external hip rotation can minimize the overlap between the femoral artery and vein [60].

\section{COMPLICATIONS AND PREVENTION}

Complications rarely arise from peripheral arterial catheterization (21 complications after 62,626 arterial cannulations $[0.03 \%]$ ), including pulseless extremities, occluded arteries, and other vascular/neurologic findings [4]. However, complications associated with arterial catheterization are common in critically ill adults [61] and children [62]. Younger children, such as neonates and infants, have a higher risk of complications [5]. The overall complication rate was $32.1 \%$ in patients aged $<2$ years. Among the complications, vasospasm and hematoma were found in $19.6 \%$ and $23.2 \%$ of patients, respectively, which could have been neglected if post-cannulation ultrasound evaluation was not done.

Furthermore, complications vary depending on the catheterization site. Recently, the overall major complication rate within 30 days was $0.034-0.2 \%$ in adults [4] and children [5], but the complication rate related to the femoral artery was $0.7-25 \%$ in children $[5,22]$. Site-specific complications are related to the anatomical characteristics of these arteries. Hence, the anatomy and relationship around the target artery must be understood well. An ultrasound-guided procedure is recommended to prevent possible complications. 


\section{Arterial occlusion or ischemia}

Arterial occlusion or ischemia following cannulation is a typical complication [42]. Permanent ischemia is rare $(0.09 \%)$, but temporary occlusion or ischemia is reported in up to $38 \%$ of cases depending on risk factors $[2,63,64]$. Therefore, caution should be exercised when patients have risk factors. Risk factors for overall vascular complications associated with catheterization in adults include the use of vasopressors, previous injury of the artery, longer duration of cannulation, hematoma, cardiac output reduction, female sex, a smaller diameter of the artery, and disseminated intravascular coagulation [63].

\section{Vasospasm}

Vasospasm can be caused by mechanical stimulation and damage to the endothelium of the vessel during arterial catheterization. This stimulation releases adenosine diphosphate and thromboxane A2, aggregating platelets and leading to vasospasm or even complete occlusion [64]. An old study reported that the incidence of vasospasm is up to $57 \%$ [2]. Vasospasm is more common in neonates or very small children because of low cardiac output, dehydration, and polycythemia [65]. Vasospasm may cause a discrepancy between peripheral arterial pressure and central arterial pressure in cardiac surgery with cardiopulmonary bypass [22], which may affect interpretation. Moreover, cannulation and catheter advancement can be disturbed by vasospasms $[9,66,67]$. Most vasospasms can be treated conservatively and completely recover. However, clinicians should always keep in mind that vasospasms can become progress to cause ischemia and necrosis.

\section{Hematoma/bleeding}

The reported incidence rates of major bleeding are $0.05 \%$ and $1.58-2.3 \%[4,64]$ for the radial and femoral arteries, respectively. Bleeding that is observed externally can be treated by applying pressure to the bleeding site directly, and thus can be easily controlled. The occurrence of hematomas related to arterial catheterization is reported to be $6.1-23.2 \%[9,60,64,68]$. Fatal hematomas, such as retroperitoneal hematomas following femoral arterial cannulation, is rare $(0.15 \%)$, but may require surgical intervention. Therefore, prevention is important. The incidence of such retroperitoneal hemorrhages can be reduced using ultra- sonography [69].

\section{Infection}

The incidence rates of arterial catheter-associated infections and sepsis are reported to be $0.024-0.38 \%$ in adults $[4,64]$ and $1.4 \%$ in children [5]. One meta-analysis revealed that the incidence of arterial catheter-related bloodstream infections was $0.7 \%$ [70]. However, the femoral artery $(0.44 \%)$ was more associated with sepsis and infection than the radial artery $(0.78 \%)$ [64]. Arterial catheter-related infections are associated with increased morbidity and mortality, and require immediate treatment [71]. Ten percent of local infections and colonization of arterial catheters can cause bacteremia, with the cause of $63 \%$ of arterial catheter-related bloodstream infections revealed be due to an extraluminal factor [72]. The risk factors related to catheter-related infections include patient factors such as chronic cardiac failure, chronic respiratory failure, immune deficiency, and diabetes, as well as procedural factors, including duration of catheter placement, bacteria-compatible catheter type, and antibiotic bonding [71].

The risk of infection when using ultrasound is controversial. In terms of reducing the attempt number and time when using ultrasound, the use of ultrasound during arterial cannulation may prevent infection [28]. On the contrary, some argue that the use of the gel for a better view of ultrasound could increase infection risk [33]. However, a recent large randomized controlled trial demonstrated that the infection risk of arterial cannulation using ultrasound did not increase compared to that without ultrasound [43].

\section{Nerve injury}

Neurological problems related to catheterization can vary depending on the site of arterial cannulation [73]. The most vulnerable nerves are the ulnar nerve around the ulnar artery, the median nerve around the brachial artery, the femoral nerve around the femoral artery, the posterior tibial nerve around the posterior tibial artery, and the deep peroneal nerve around the dorsalis pedis artery. These nerves can be easily injured directly by the needle or the pressure following hematoma, pseudoaneurysm or fluid extravasation, and chemical injury [74]. The reported incidence of nerve injury varies, but median nerve damage related to brachial arterial cannulation is most common at $9 \%$, followed by femoral nerve injury [73]. With ultrasound 
guidance, the anatomical relationship between the target artery and adjacent structures can be evaluated before cannulation, and the direction of the needle can be adjusted to avoid direct trauma to the nerve during arterial catheterization [73].

\section{CONCLUSION}

Ultrasound-guided arterial catheterization is more effective than conventional methods in terms of success rate and complications. For successful ultrasound-guided arterial catheterization, it is necessary to select an appropriate artery and catheter, perform an accurate ultrasound-guided procedure, and apply useful techniques to increase success rates. Practitioners should become familiar with vascular anatomy and ultrasound-guided arterial catheterization techniques.

\section{CONFLICTS OF INTEREST}

No potential conflict of interest relevant to this article was reported.

\section{DATA AVAILABILITY STATEMENT}

Data sharing not applicable to this article as no datasets were generated or analyzed during the current study (review article).

\section{AUTHOR CONTRIBUTIONS}

Conceptualization: Jin-Tae Kim. Project administration: Jin-Tae Kim. Visualization: Young-Eun Jang. Investigation: Sang-Hwan Ji, Eun-Hee Kim, Ji-Hyun Lee, Hee-Soo Kim. Software: Sang-Hwan Ji. Supervision: Jin-Tae Kim. Validation: Ji-Hyun Lee. Writing -original draft: Sung-Ae Cho, Young-Eun Jang, Jin-Tae Kim. Writing - review \& editing: Sung-Ae Cho, Young-Eun Jang, Hee-Soo Kim, Jin-Tae Kim.

\section{ORCID}

Sung-Ae Cho, https://orcid.org/0000-0002-1519-3787 Young-Eun Jang, https://orcid.org/0000-0002-7511-4104 Sang-Hwan Ji, https://orcid.org/0000-0001-6736-4464 Eun-Hee Kim, https://orcid.org/0000-0003-0697-1935 Ji-Hyun Lee, https://orcid.org/0000-0002-8384-8191 Hee-Soo Kim, https://orcid.org/0000-0002-2661-7944
Jin-Tae Kim, https://orcid.org/0000-0002-3738-0081

\section{REFERENCES}

1. Ishii S, Shime N, Shibasaki M, Sawa T. Ultrasound-guided radial artery catheterization in infants and small children. Pediatr Crit Care Med 2013; 14: 471-3.

2. Kim JM, Arakawa K, Bliss J. Arterial cannulation: factors in the development of occlusion. Anesth Analg 1975; 54: 836-41.

3. Latham GJ, Bosenberg AT, Low DK. Images in anesthesiology: radial artery spasm in an infant as documented by high-frequency micro-ultrasound. Anesthesiology 2014; 120: 1254.

4. Nuttall G, Burckhardt J, Hadley A, Kane S, Kor D, Marienau MS, et al. Surgical and patient risk factors for severe arterial line complications in adults. Anesthesiology 2016; 124: 590-7.

5. Gleich SJ, Wong AV, Handlogten KS, Thum DE, Nemergut ME. Major short-term complications of arterial cannulation for monitoring in children. Anesthesiology 2021; 134: 26-34.

6. Beniwal S, Bhargava K, Kausik SK. Size of distal radial and distal ulnar arteries in adults of southern Rajasthan and their implications for percutaneous coronary interventions. Indian Heart J 2014; 66: 506-9.

7. Ashraf T, Panhwar Z, Habib S, Memon MA, Shamsi F, Arif J. Size of radial and ulnar artery in local population. J Pak Med Assoc 2010; 60: 817-9.

8. Kim EH, Lee JH, Song IK, Kim JT, Lee WJ, Kim HS. Posterior tibial artery as an alternative to the radial artery for arterial cannulation site in small children: a randomized controlled study. Anesthesiology 2017; 127: 423-31.

9. Jang YE, Ji SH, Kim EH, Lee JH, Kim HS, Mossad EB, et al. Subcutaneous nitroglycerin for radial arterial catheterization in pediatric patients: a randomized controlled trial. Anesthesiology 2020; 133: 53-63.

10. Min SW, Cho HR, Jeon YT, Oh AY, Park HP, Yang CW, et al. Effect of bevel direction on the success rate of ultrasound-guided radial arterial catheterization. BMC Anesthesiol 2016; 16: 34.

11. Tiru B, Bloomstone JA, McGee WT. Radial artery cannulation: a review article. J Anesth Clin Res 2012; 3: 209.

12. Lamperti M, Biasucci DG, Disma N, Pittiruti M, Breschan C, Vailati D, et al. European Society of Anaesthesiology guidelines on peri-operative use of ultrasound-guided for vascular access (PERSEUS vascular access). Eur J Anaesthesiol 2020; 37: 34476.

13. Bertrand OF, Carey PC, Gilchrist IC. Allen or no allen: that is the question! J Am Coll Cardiol 2014; 63: 1842-4.

14. Riekkinen HV, Karkola KO, Kankainen A. The radial artery is larger than the ulnar. Ann Thorac Surg 2003; 75: 882-4. 
15. Schindler E, Kowald B, Suess H, Niehaus-Borquez B, Tausch B, Brecher A. Catheterization of the radial or brachial artery in neonates and infants. Paediatr Anaesth 2005; 15: 677-82.

16. Tomiyama Y, Yoshinaga K, Fujii S, Ochi N, Inoue M, Nishida M, et al. Accurate quantitative measurements of brachial artery cross-sectional vascular area and vascular volume elastic modulus using automated oscillometric measurements: comparison with brachial artery ultrasound. Hypertens Res 2015; 38: 478-84.

17. Kim EH, Lee HC, Chung J, Ji SH, Jang YE, Lee JH, et al. Flow-mediated dilatation of the brachial artery for assessing endothelial dysfunction in children with Moyamoya disease. Pediatr Neurosurg 2020; 55: 149-54.

18. Khan ZA, Khan MA, MohammednourAltaf F, Alkhushi AG, Alasmari WA. Diameter of the dorsalis pedis artery and its clinical relevance. IOSR-JDMS 2016; 15: 129-33.

19. Babuccu O, Ozdemir H, Hosnuter M, Kargi E, Söğüt A, Ayoglu FN. Cross-sectional internal diameters of radial, thoracodorsal, and dorsalis pedis arteries in children: relationship to subject sex, age, and body size. J Reconstr Microsurg 2006; 22: 49-52.

20. Lorbeer R, Grotz A, Dörr M, Völzke H, Lieb W, Kühn JP, et al. Reference values of vessel diameters, stenosis prevalence, and arterial variations of the lower limb arteries in a male population sample using contrast-enhanced MR angiography. PLoS One 2018; 13: e0197559.

21. Sun J, Ding Z, Qian Y, Peng YG. Central-radial artery pressure gradient after cardiopulmonary bypass is associated with cardiac function and may affect therapeutic direction. PLoS One 2013; 8: e68890.

22. Cho HJ, Lee SH, Jeong IS, Yoon NS, Ma JS, Ahn BH. Differences in perioperative femoral and radial arterial blood pressure in neonates and infants undergoing cardiac surgery requiring cardiopulmonary bypass. J Pediatr (Rio J) 2018; 94: 76-81.

23. Rudnick MR, Marchi LD, Plotkin JS. Hemodynamic monitoring during liver transplantation: a state of the art review. World J Hepatol 2015; 7: 1302-11.

24. Spector KS, Lawson WE. Optimizing safe femoral access during cardiac catheterization. Catheter Cardiovasc Interv 2001; 53: 209-12.

25. Bhatia N, Sivaprakasam J, Allford M, Guruswamy V. The relative position of femoral artery and vein in children under general anesthesia--an ultrasound-guided observational study. Paediatr Anaesth 2014; 24: 1164-8.

26. Rajebi H, Rajebi MR. Optimizing common femoral artery access. Tech Vasc Interv Radiol 2015; 18: 76-81.

27. Troianos CA, Hartman GS, Glas KE, Skubas NJ, Eberhardt RT, Walker JD, et al. Councils on Intraoperative Echocardiography and Vascular Ultrasound of the American Society of Echocardiography. Guidelines for performing ultrasound guided vascular cannulation: recommendations of the American Society of Echocardiography and the Society of Cardiovascular Anesthesiologists. J Am Soc Echocardiogr 2011; 24: 1291-318.

28. White L, Halpin A, Turner M, Wallace L. Ultrasound-guided radial artery cannulation in adult and paediatric populations: a systematic review and meta-analysis. Br J Anaesth 2016; 116: 610-7.

29. Seto AH, Roberts JS, Abu-Fadel MS, Czak SJ, Latif F, Jain SP, et al. Real-time ultrasound guidance facilitates transradial access: RAUST (radial artery access with ultrasound trial). JACC Cardiovasc Interv 2015; 8: 283-91.

30. Jang YE, Kim EH, Lee JH, Kim HS, Kim JT. Guidewire-assisted vs. direct radial arterial cannulation in neonates and infants: a randomised controlled trial. Eur J Anaesthesiol 2019; 36: 73844.

31. Abu-Omar Y, Mussa S, Anastasiadis K, Steel S, Hands L, Taggart DP. Duplex ultrasonography predicts safety of radial artery harvest in the presence of an abnormal Allen test. Ann Thorac Surg 2004; 77: 116-9.

32. Habib J, Baetz L, Satiani B. Assessment of collateral circulation to the hand prior to radial artery harvest. Vasc Med 2012; 17: 352-61.

33. Chittick P, Russo V, Sims M, Robinson-Dunn B, Oleszkowicz S, Sawarynski K, et al. An outbreak of Pseudomonas aeruginosa respiratory tract infections associated with intrinsically contaminated ultrasound transmission gel. Infect Control Hosp Epidemiol 2013; 34: 850-3.

34. Berk D, Gurkan Y, Kus A, Ulugol H, Solak M, Toker K. Ultrasound-guided radial arterial cannulation: long axis/in-plane versus short axis/out-of-plane approaches? J Clin Monit Comput 2013; 27: 319-24.

35. Quan Z, Tian M, Chi P, Cao Y, Li X, Peng K. Modified short-axis out-of-plane ultrasound versus conventional long-axis inplane ultrasound to guide radial artery cannulation: a randomized controlled trial. Anesth Analg 2014; 119: 163-9.

36. Sethi S, Maitra S, Saini V, Samra T, Malhotra SK. Comparison of short-axis out-of-plane versus long-axis in-plane ultrasound-guided radial arterial cannulation in adult patients: a randomized controlled trial. J Anesth 2017; 31: 89-94.

37. Song IK, Choi JY, Lee JH, Kim EH, Kim HJ, Kim HS, et al. Short-axis/out-of-plane or long-axis/in-plane ultrasound-guided arterial cannulation in children: a randomised controlled trial. Eur J Anaesthesiol 2016; 33: 522-7.

38. Kiberenge RK, Ueda K, Rosauer B. Ultrasound-guided dynamic needle tip positioning technique versus palpation technique 
for radial arterial cannulation in adult surgical patients: a randomized controlled trial. Anesth Analg 2018; 126: 120-6.

39. Liu L, Tan Y, Li S, Tian J. "Modified dynamic needle tip positioning" short-axis, out-of-plane, ultrasound-guided radial artery cannulation in neonates: a randomized controlled trial. Anesth Analg 2019; 129: 178-83.

40. Nam K, Jeon Y, Yoon S, Kwon SM, Kang P, Cho YJ, et al. Ultrasound-guided radial artery cannulation using dynamic needle tip positioning versus conventional long-axis in-plane techniques in cardiac surgery patients: a randomized, controlled trial. Minerva Anestesiol 2020; 86: 30-7.

41. Quan Z, Zhang L, Zhou C, Chi P, He H, Li Y. Acoustic shadowing facilitates ultrasound-guided radial artery cannulation in young children. Anesthesiology 2019; 131: 1018-24.

42. Evans PJ, Kerr JH. Arterial occlusion after cannulation. Br Med J 1975; 3: 197.

43. Buetti N, Ruckly S, Lucet JC, Bouadma L, Schwebel C, Mimoz O, et al. Ultrasound guidance and risk for intravascular catheter-related infections among peripheral arterial catheters: a post-hoc analysis of two large randomized-controlled trials. Ann Intensive Care 2020; 10: 89.

44. Pancholy SB, Coppola J, Patel T. Subcutaneous administration of nitroglycerin to facilitate radial artery cannulation. Catheter Cardiovasc Interv 2006; 68: 389-91.

45. Candemir B, Kumbasar D, Turhan S, Kilickap M, Ozdol C, Akyurek O, et al. Facilitation of radial artery cannulation by periradial subcutaneous administration of nitroglycerin. J Vasc Interv Radiol 2009; 20: 1151-6.

46. Ezhumalai B, Satheesh S, Jayaraman B. Effects of subcutaneously infiltrated nitroglycerin on diameter, palpability, ease-ofpuncture and pre-cannulation spasm of radial artery during transradial coronary angiography. Indian Heart J 2014; 66: 5937.

47. Chen Y, Ke Z, Xiao J, Lin M, Huang X, Yan C, et al. Subcutaneous injection of nitroglycerin at the radial artery puncture site reduces the risk of early radial artery occlusion after transradial coronary catheterization: a randomized, placebo- controlled clinical trial. Circ Cardiovasc Interv 2018; 11: e006571.

48. Coppola J, Patel T, Kwan T, Sanghvi K, Srivastava S, Shah S, et al. Nitroglycerin, nitroprusside, or both, in preventing radial artery spasm during transradial artery catheterization. J Invasive Cardiol 2006; 18: 155-8.

49. Dharma S, Kedev S, Patel T, Kiemeneij F, Gilchrist IC. A novel approach to reduce radial artery occlusion after transradial catheterization: postprocedural/prehemostasis intra-arterial nitroglycerin. Catheter Cardiovasc Interv 2015; 85: 818-25.

50. Majure DT, Hallaux M, Yeghiazarians Y, Boyle AJ. Topical nitro- glycerin and lidocaine locally vasodilate the radial artery without affecting systemic blood pressure: a dose-finding phase I study. J Crit Care 2012; 27: 532.e9-13.

51. Beyer AT, Ng R, Singh A, Zimmet J, Shunk K, Yeghiazarians Y, et al. Topical nitroglycerin and lidocaine to dilate the radial artery prior to transradial cardiac catheterization: a randomized, placebo-controlled, double-blind clinical trial: the PRE-DILATE Study. Int J Cardiol 2013; 168: 2575-8.

52. Reusz G, Langer C, Jakab L, Morvay Z. Ultrasound-guided vascular access: the importance of the needle bevel. Can J Anaesth 2012; 59: 499-500.

53. Beards SC, Doedens L, Jackson A, Lipman J. A comparison of arterial lines and insertion techniques in critically ill patients. Anaesthesia 1994; 49: 968-73.

54. Yildirim V, Ozal E, Cosar A, Bolcal C, Acikel CH, Kiliç S, et al. Direct versus guidewire-assisted pediatric radial artery cannulation technique. J Cardiothorac Vasc Anesth 2006; 20: 48-50.

55. Lavallée C, Ayoub C, Mansour A, Lambert J, Lebon JS, Lalu MM, et al. Subclavian and axillary vessel anatomy: a prospective observational ultrasound study. Can J Anaesth 2018; 65: 350-9.

56. Melhuish TM, White LD. Optimal wrist positioning for radial arterial cannulation in adults: a systematic review and meta-analysis. Am J Emerg Med 2016; 34: 2372-8.

57. Blanco P. Ultrasound-guided vascular cannulation in critical care patients: a practical review. Med Intensiva 2016; 40: 560 71.

58. Werner SL, Jones RA, Emerman CL. Effect of hip abduction and external rotation on femoral vein exposure for possible cannulation. J Emerg Med 2008; 35: 73-5.

59. Ahn HJ, Lee JW, Yoo SW, Kim JH, Kim KD, Yoo IS, et al. Novel body positioning maximizes femoral venous size in adults: an ultrasonographic evaluation. Hong Kong J Emerg Med 2018; 25: $338-42$.

60. Dahl MR, Smead WL, McSweeney TD. Radial artery cannulation: a comparison of 15.2- and 4.45-cm catheters. J Clin Monit 1992; 8: 193-7.

61. Belda FJ, Aguilar G, Teboul JL, Pestaña D, Redondo FJ, Malbrain $\mathrm{M}$, et al. PICS Investigators Group. Complications related to less-invasive haemodynamic monitoring. Br J Anaesth 2011; 106: 482-6.

62. King MA, Garrison MM, Vavilala MS, Zimmerman JJ, Rivara FP, Complications associated with arterial catheterization in children. Pediatr Crit Care Med 2008; 9: 367-71.

63. Lipira AB, Mackinnon SE, Fox IK. Axillary arterial catheter use associated with hand ischemia in a multi-trauma patient: case report and literature review. J Clin Anesth 2011; 23: 325-8. 
64. Scheer B, Perel A, Pfeiffer UJ. Clinical review: complications and risk factors of peripheral arterial catheters used for haemodynamic monitoring in anaesthesia and intensive care medicine. Crit Care 2002; 6: 199-204.

65. Baserga MC, Puri A, Sola A. The use of topical nitroglycerin ointment to treat peripheral tissue ischemia secondary to arterial line complications in neonates. J Perinatol 2002; 22: 416-9.

66. Cuper NJ, de Graaff JC, Hartman BJ, Verdaasdonk RM, Kalkman CJ. Difficult arterial cannulation in children: is a near-infrared vascular imaging system the answer? Br J Anaesth 2012; 109: 420-6.

67. Ho HH, Jafary FH, Ong PJ. Radial artery spasm during transradial cardiac catheterization and percutaneous coronary intervention: incidence, predisposing factors, prevention, and management. Cardiovasc Revasc Med 2012; 13: 193-5.

68. Puri VK, Carlson RW, Bander JJ, Weil MH. Complications of vascular catheterization in the critically ill. A prospective study. Crit Care Med 1980; 8: 495-9.
69. Wang A, Hendin A, Millington SJ, Koenig S, Eisen LA, Shiloh AL. Better with ultrasound: arterial line placement. Chest 2020; 157: 574-9.

70. O'Horo JC, Maki DG, Krupp AE, Safdar N. Arterial catheters as a source of bloodstream infection: a systematic review and meta-analysis. Crit Care Med 2014; 42: 1334-9.

71. Hambsch ZJ, Kerfeld MJ, Kirkpatrick DR, McEntire DM, Reisbig MD, Youngblood CF, et al. Arterial catheterization and infection: toll-like receptors in defense against microorganisms and therapeutic implications. Clin Transl Sci 2015; 8: 857-70.

72. Safdar N, O'Horo JC, Maki DG. Arterial catheter-related bloodstream infection: incidence, pathogenesis, risk factors and prevention. J Hosp Infect 2013; 85: 189-95.

73. Kuo F, Park J, Chow K, Chen A, Walsworth MK. Avoiding peripheral nerve injury in arterial interventions. Diagn Interv Radiol 2019; 25: 380-91.

74. Rubinos C, Ruland S. Neurologic complications in the intensive care unit. Curr Neurol Neurosci Rep 2016; 16: 57. 